\title{
Haltung, Fütterung und häufigste Erkrankungen der Ratte
}

\author{
Anja Ewringmann
}

Ratten (Rattus norwegicus) sind als Heimtiere äußerst beliebt und werden oft in der tierärztlichen Praxis vorgestellt. Sie sind sehr robuste Tiere, sodass haltungsund fütterungsbedingte Erkrankungen nur sehr selten vorkommen. Dennoch sind eine artgerechte Unterbringung und Fütterung unter Tierschutzaspekten absolut wünschenswert, zumal Mängel Immunsuppressionen und damit sekundäre Erkrankungen begünstigen.

\section{Biologie}

Während wildlebende Ratten eher dämmerungs- und nachtaktiv sind, haben sich die domestizierten Tiere an die Lebensart ihrer Besitzer angepasst. Schlafund Wachphasen wechseln sich über den gesamten Tagesverlauf ab. Die Lebenserwartung der Ratte beträgt etwa 1,5-3 Jahre. Geschlechtsreif werden die Tiere mit ca. 5-6 Wochen. Sie sind ganzjährig polyöstrisch mit einem Brunstzyklus, der nur 4-5 Tage dauert. Nach einer Trächtigkeitsdauer von 21-24 Tagen werden 4-16 Jungtiere geboren, die von der Mutter 3 Wochen lang gesäugt werden.

\section{Haltung \\ Gruppenzusammensetzung}

Ratten leben in der Natur in großen Kolonien zusammen. Daher sollten auch in der Heimtierhaltung mindestens 2 Tiere oder Kleingruppen von 4-6 Tieren gepflegt werden. Dabei kann es sich sowohl um gleichgeschlechtliche Gruppen als auch um Gruppen von Weibchen mit männlichen Kastraten handeln. Bei einer Paarhaltung ist es sinnvoll, dass beide Tiere in etwa gleich alt sind, damit sie sich bezüglich ihrer Bedürfnisse (längere Schlaf- und Ruhephasen bei alten Ratten, ausgeprägtes Spielverhalten bei jungen Tieren) ergänzen. In größeren Gruppen kann das Altersspektrum breiter gestreut sein. Wichtig ist jedoch auch hier, dass aus verschiedenen Altersklassen mindestens 2 Tiere vorhanden sind. Andernfalls sind alte Tiere schnell überbzw. junge Ratten unterfordert.

Soll eine Vergesellschaftung stattfinden, sollte diese möglichst auf neutralem Boden erfolgen. Meist ist es einfacher, in eine Gruppe, die aus alten Ratten besteht, Jungtiere (mindestens 2!) zu integrieren, da sich diese schneller unterordnen.

\section{Unterbringung}

Ein Rattenkäfig sollte so groß wie möglich sein, auch wenn den Tieren regelmäßig Freilauf gewährt wird. Als Grundfläche für ein Paar darf ein Maß von $100 \times 60 \mathrm{~cm}$ bei einer Höhe von $100 \mathrm{~cm}$ nicht unterschritten werden. Für die Gruppenhaltung eignen sich größere Zimmervolieren oder Eigenbauten ( Abb.1). Der Käfig muss mit mehreren Etagen sowie Kletter- und Versteckmöglichkeiten eingerichtet sein ( Abb.2). Etagen und Häuschen sollten aus Holz bestehen, als Klettermöglichkeiten bieten sich neben Ästen ( $\triangleright$ Abb. 3 ) auch Röhren (z. B. aus Kork, geflochtenem Heu, Pappe), Körbe und dicke Seile an. Hängematten als Aussichts- und Ruheplätze werden ebenfalls gerne angenommen.

Als Einstreu eignen sich geschreddertes Zeitungspapier, ungebleichter Zellstoff oder Baumwollstreu.

\section{Die handelsübliche Kleintierstreu sollte in der Rattenhaltung nicht verwendet werden, da Atemwegserkrankungen, die in Rattenbeständen ohnehin weit ver- breitet sind, durch die starke Staub- entwicklung begünstigt werden.}

Auch als Nistmaterial sind staubfreie Materialien (z.B. Baumwollstreu) vorzuziehen.

Zusätzliche Beschäftigung bieten „Buddelkisten“, die z.B. mit Sand und darin versteckten Leckerbissen zeitweise zur Verfügung gestellt werden können. Auch hier muss bei Tieren mit Atemwegserkrankungen gegebenenfalls auf staubfreie Streu, z. B. Papierschnipsel, zurückgegriffen werden.

\section{Freilauf}

Ratten müssen täglich ausreichend Freilauf erhalten. Dieser erfolgt unter Aufsicht in einem „rattensicher“ gestalteten Zimmer ohne Zugriff auf Stromkabel oder Giftpflanzen (wie z.B. Oleander, Alpenveilchen und Yucca-Palme).

Bei zu geringem Platz- und Beschäftigungsangebot können sich schnell Aggressionen (gegenüber Partnertieren und dem Menschen) sowie Revierstreitigkeiten entwickeln, die mitunter in Beißereien münden ( $\bullet$ Abb.4).

\section{Fütterung}

Ratten sind Allesfresser mit einem Schwerpunkt auf einer granivoren $\mathrm{Er}$ nährung. Rohfaser kann von den Tieren nur begrenzt aufgeschlossen werden, sodass Rohfasergehalte von $10 \%$ in der Ration nicht überschritten werden sollten.

Die Tiere nehmen im Laufe des Tages viele kleine Mahlzeiten zu sich und verbringen viel Zeit mit der Nahrungssuche.

\section{.konkret}

Trockenfutter sollte im Käfig verteilt und nicht ausschließlich in Näpfen angeboten werden. 
Als Grundnahrung dient eine Futtermischung, die aus verschiedenen Getreidearten und Sämereien besteht. Nüsse oder andere stark fetthaltige Bestandteile (z.B. Kürbis- und Sonnenblumenkerne, Hirse) dürfen in dieser Mischung nur in geringen Mengen enthalten sein oder sollten als gelegentlicher Leckerbissen angeboten werden. Bei überhöhter Zufuhr entsteht eine Adipositas, die Folgeerkrankungen wie z.B. Pododermatitis ( $\mathbf{A b b}$.5), Leberverfettung oder degenerative Erkrankungen des Bewegungsapparates nach sich zieht.

Das Grundfutter wird zudem durch getrocknetes Gemüse und Trockenkräuter ergänzt. Qualitativ hochwertiges, staubarmes Heu kann in kleinen Portionen zur Deckung des Rohfaserbedarfes angeboten werden. Tiere mit Atemwegserkrankungen sollten stattdessen Heucobs oder Kräuterpellets bekommen. Zusätzlich sollten Ratten 1- bis 2-mal täglich Frischfutter erhalten. Geeignete Gemüsesorten sind z.B.:
- Karotten
- Zucchini
- Gurken
- Paprika

Auch kleine Mengen Salat und frische Kräuter können angeboten werden.

Gern werden auch Obstsorten wie z. B.:
- Apfel
- Melone
- Banane
- Weintraube
- Birne
angenommen.

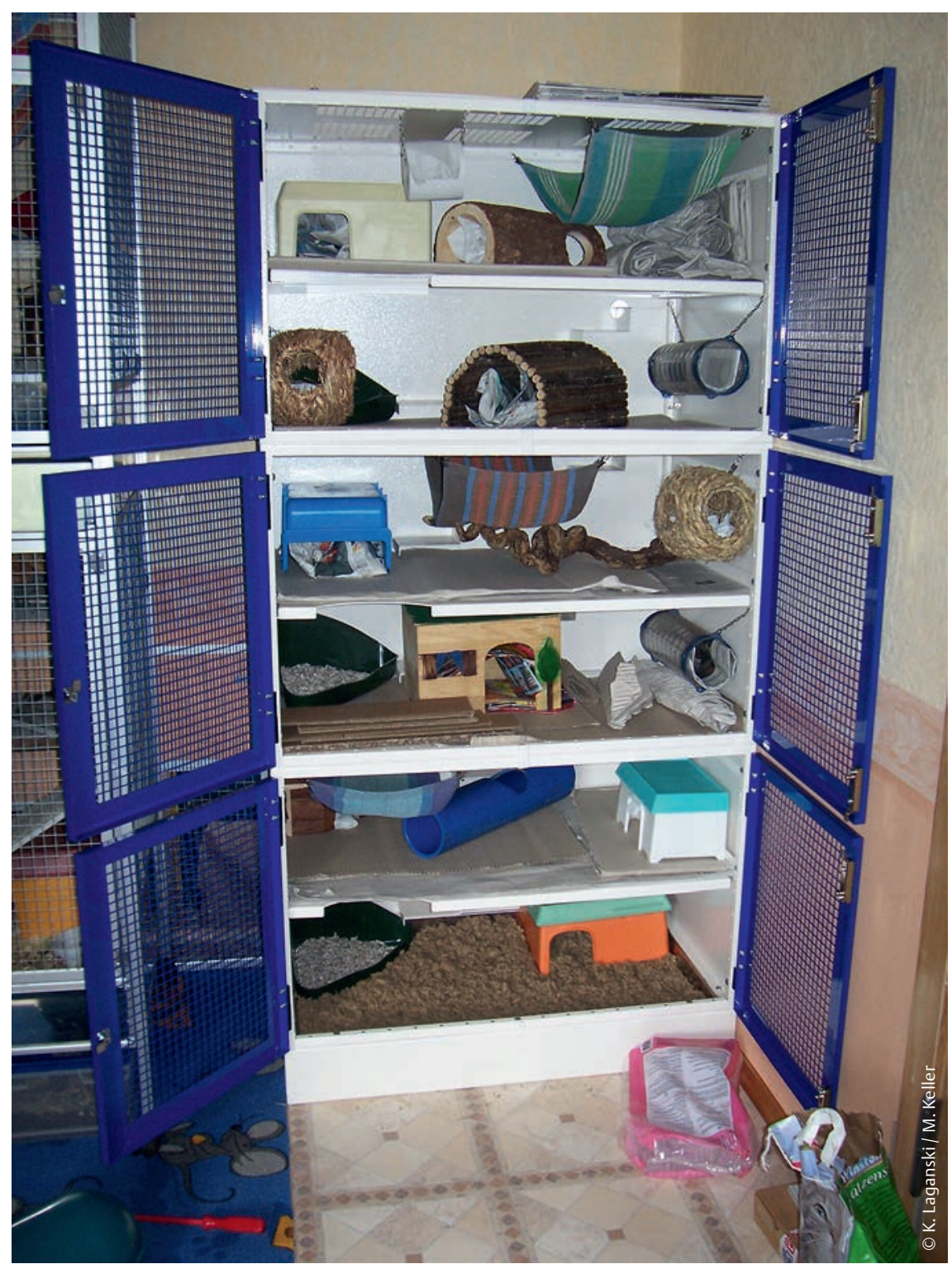

Abb. 1 Eigenbau für Ratten.

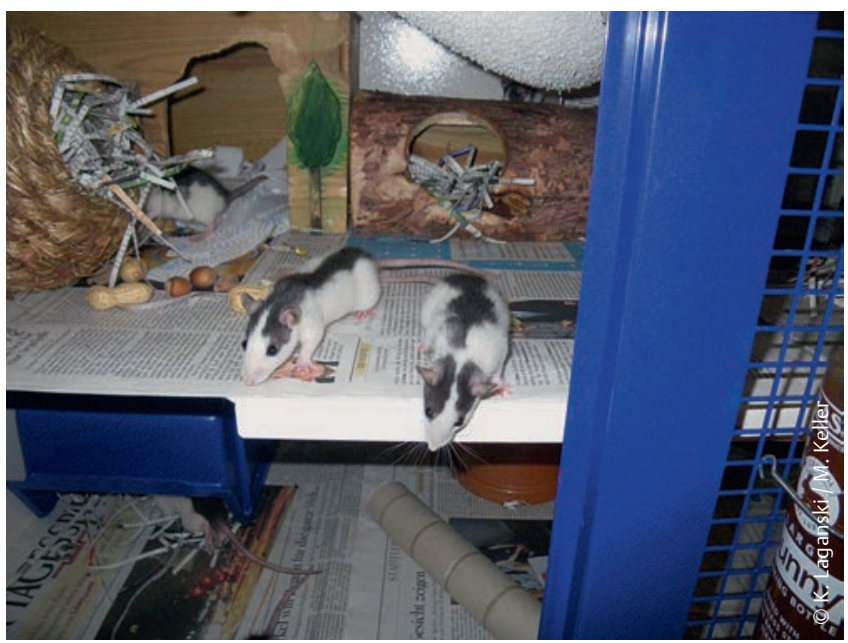

Abb. 2 Artgerecht eingerichtetes „Rattenheim“.

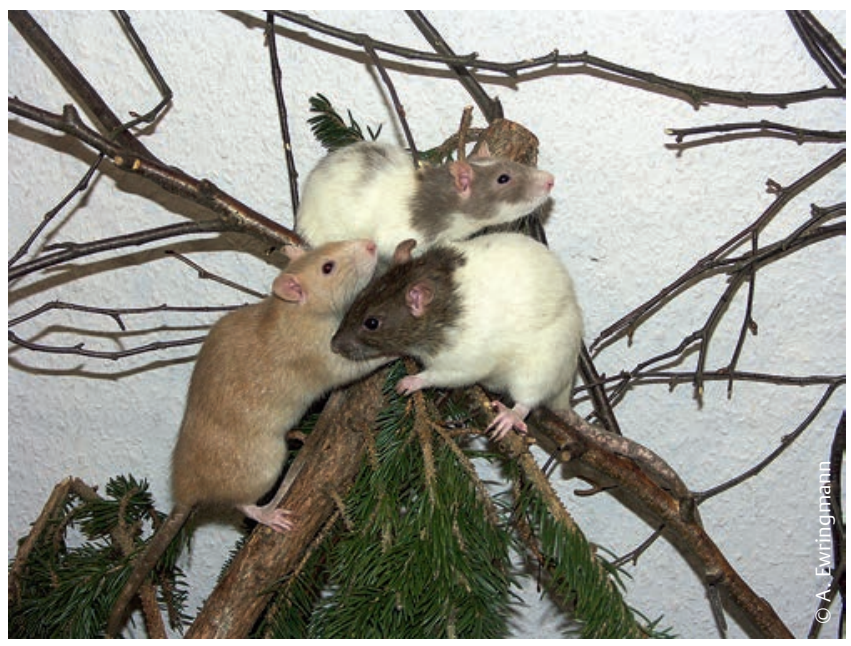

Abb. 3 Äste dienen zum Klettern. 
Als Leckerbissen können gelegentlich in kleinen Mengen Mais, Nüsse (in der Schale), gekochte Kartoffeln, Reis oder Nudeln sowie getrocknetes Brot angeboten werden. Um das Nagebedürfnis der Ratten zu befriedigen, können sie zusätzlich zur benagbaren Käfigeinrichtung frische Zweige unbehandelter Obstbäume, Haselnusssträucher oder Weiden erhalten.

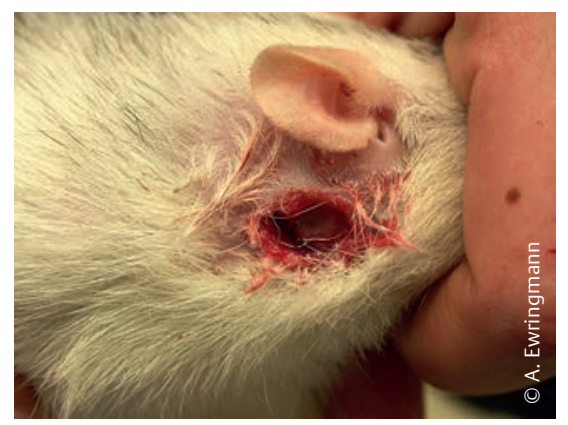

Abb. 4 Abszedierte Bissverletzung am Hals nach Rangordnungskampf.

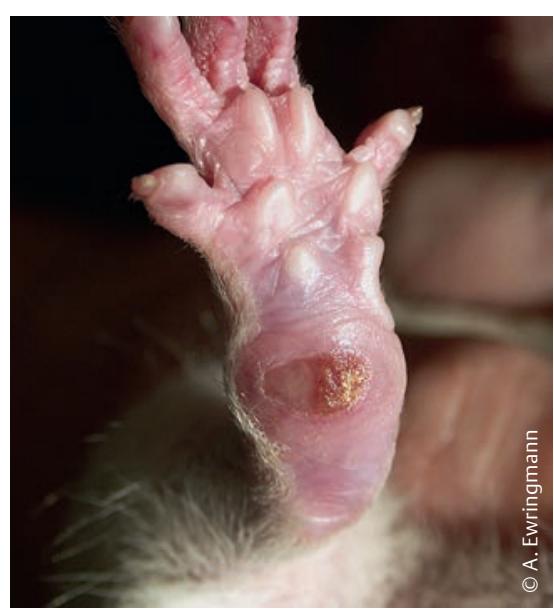

Abb. 5 Pododermatitis. Bei Adipositas steigt die Druckbelastung auf die Ballen.

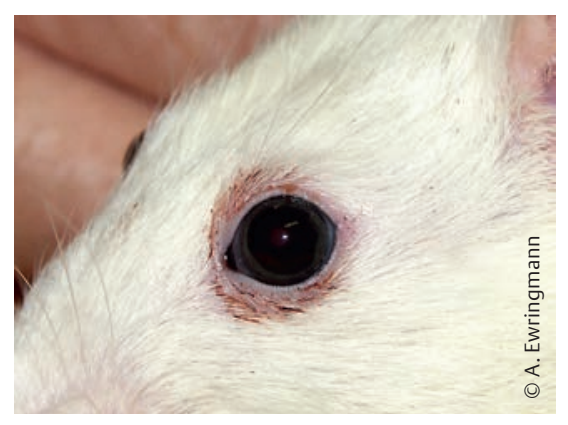

Abb. 6 Die Ansammlung von Sekret der Harderschen Drüsen um das Auge (Brillenbildung) ist Anzeichen für ein reduziertes Putzverhalten, nicht jedoch spezifisch für eine bestimmte Erkrankung.
Ein weiterer Baustein einer ausgewogenen Ernährung ist das tierische Eiweiß. Um die Versorgung adulter Tiere zu sichern, sollten 1- bis 2-mal wöchentlich ungezuckerter Magerquark oder -joghurt, hart gekochtes Ei oder milder Käse angeboten werden. Auch vitaminreich gefütterte Mehlwürmer, Hundekuchen oder Katzentrockenfutter sind geeignet. Der Bedarf ist bei Jungtieren sowie trächtigen und säugenden Muttertieren deutlich höher als bei Ratten in anderen Lebensphasen.

Frisches Wasser muss stets angeboten werden und sollte in einer hygienischen Trinkflasche zur Verfügung stehen.

\section{Zwangsfütterung}

Zur Zwangsernährung von Ratten sind die im Handel befindlichen Produkte für herbivore Tierarten (z.B. Critical Care, RodiCare $^{\circledR}$ instant, Herbi Care plus) als alleinige Nahrung nicht geeignet! Hier muss stattdessen ein Brei hergestellt werden. Dieser besteht aus:

- aufgelösten Schmelzflocken

- Obst- oder Gemüsebrei (aus dem Gläschen)

- etwas Magerquark oder -joghurt

- etwas Vitaminpaste für Katzen (z.B. Nutri Cal ${ }^{\circledR}$ )

Zur Rohfaserergänzung kann zudem eine Prise eines der oben genannten Fertigfuttermittel beigesetzt werden.

\section{Häufige Erkrankungen Mykoplasmose}

Der Erreger der Mykoplasmose, Mycoplasma pulmonis, ist in Rattenbeständen weit verbreitet. Die Übertragung erfolgt durch direkten Kontakt, Aerosole, den Deckakt und intrauterin. Auch eine Infektion über belebte und unbelebte Vektoren ist möglich.

Der Erreger besiedelt v.a. die Schleimhäute des Atmungstrakts und verursacht chronische und progressiv verlaufende Erkrankungen, bei denen es zu zunehmender Schädigung und zum fortschreitenden Umbau des respiratorischen Epithels kommt.
Mykoplasmosen verlaufen oft über lange Zeit latent. Ein Ausbruch wird durch Immunsuppression begünstigt. Mögliche immunsupprimierende Faktoren sind z.B.: - Stress

- Rangordnungskämpfe

- Umgebungswechsel

- Veränderungen der Gruppenzusammensetzung

- unzureichende Haltungs- und Fütterungbedingungen

- fortgeschrittenes Alter

- andere Grunderkrankungen (z.B. Endoparasitosen)

Begünstigend für Erkrankungsausbrüche sind zudem Reizungen der Atemwege (z.B. durch staubende Einstreu), die bakterielle Sekundärinfektionen fördern (z.B. mit Pasteurella pneumotropica, Bordetella bronchiseptica, Corynebacterium kutscheri und Streptococcus pneumoniae).

\section{Symptomatik}

Das erste Symptom einer Mykoplasmose ist meist gelegentliches Niesen, das sich progressiv intensiviert. Durch die Erregeransiedlung in tieferen Bereichen des Respirationstrakts entstehen Bronchopneumonien, die mit knarrenden oder knackenden Atemgeräuschen und deutlich pumpender Flankenatmung einhergehen. Nasen- und Augenausfluss fehlen meist, können durch bakterielle Sekundärinfektionen jedoch hinzutreten. Das Allgemeinbefinden der Tiere verschlechtert sich zusehends. Aufgrund der Atemnot ist eine Nahrungsaufnahme immer weniger möglich und es kommt zu fortschreitender Abmagerung. Da sich die Tiere nicht mehr putzen, wirkt das Fell ungepflegt und struppig. Das rötliche Sekret der Harderschen Drüsen sammelt sich um die Augen und führt zur sog. Brillenbildung ( $\triangleright$ Abb.6). Durch Abfluss über den Tränennasengang sind auch die Nasenöffnungen rötlich verkrustet. 
Mögliche Komplikationen, die durch die Erregerabsiedlung in andere Organe verursacht werden, sind:

- Otitiden mit plötzlicher Kopfschiefhaltung und Manegebewegungen ( Abb. 7 und $>$ Abb. 8 )

- Arthritiden mit schweren Bewegungsstörungen

- Genitalinfektionen mit Aborten und Unfruchtbarkeit

In fortgeschrittenen Mykoplasmosefällen kann es zum Spontanpneumothorax kommen ( $\triangleright$ Abb.9).

\section{Diagnostik}

Eine exakte Diagnosesicherung am lebenden Tier ist i.d.R. nicht möglich, da der Erreger im Bereich der Nasenhöhlen und der tieferen Atemwege lokalisiert ist, die einer Tupferprobenentnahme

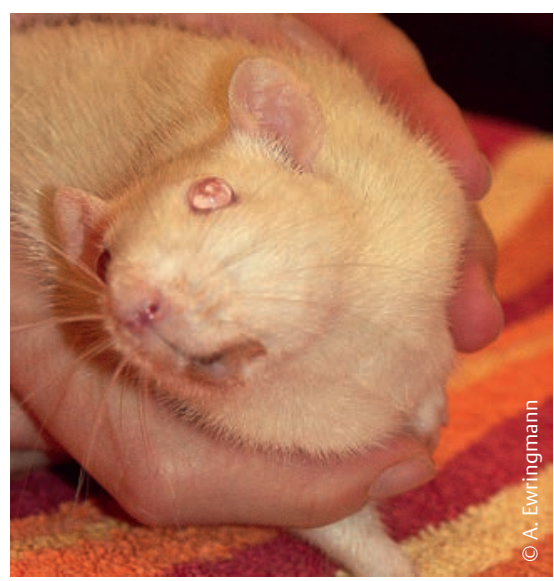

Abb. 7 Kopfschiefhaltung durch Otitis media.

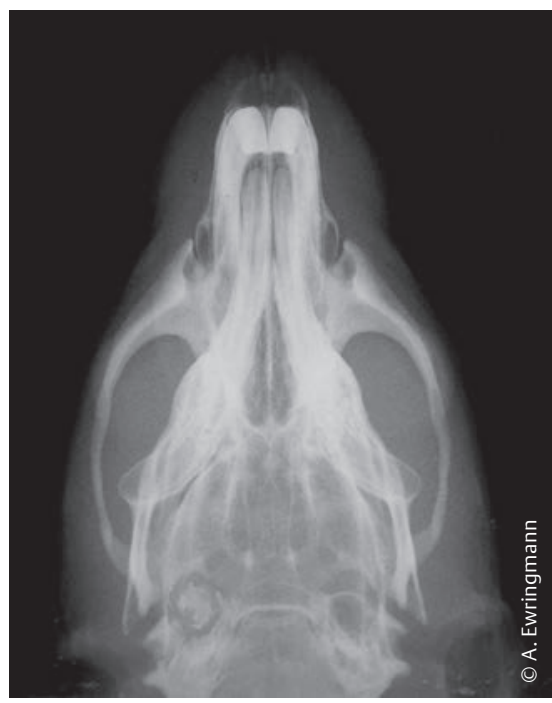

Abb. 8 Linksseitige Bullaverschattung bei Otitis media/interna. nicht zugänglich sind. Muss ein Patient aus einem Bestand euthanasiert werden, sollten jedoch aus den genannten Lokalisationen Proben (Abstriche, Gewebeproben) entnommen und zur Untersuchung eingesandt werden, damit eine gezielte Behandlung der verbliebenen Tiere möglich wird.

\section{Therapie}

Die Mykoplasmose ist eine chronische Erkrankung und es besteht keine Möglichkeit einer Erregerelimination und Ausheilung. Durch eine Behandlung können lediglich die Symptome gemildert und das Fortschreiten der Erkrankung verzögert werden.

Im Anfangsstadium erfolgt eine Behandlung zunächst mit Mukolytika (z.B. Acetylcystein, 3-5 mg/kg 2-mal tgl. p.o. oder s.c.; Bromhexin, 0,5 mg 2-mal tgl. p.o. oder s.c.) und Paramunitätsinducern (z.B. Zylexis ${ }^{\circledR}, 0,5 \mathrm{ml} /$ Tier am 1., 2. und 7. Behandlungstag). Zudem können Inhalationen mit Thymian- oder Salbeitee durchgeführt werden, um Schleim in den Atemwegen zu lösen. Alternativ eignen sich auch Injektionslösungen von Mukolytika zur Inhalation. Diese werden mit isotoner Kochsalzlösung verdünnt und können dann über einen Kaltvernebler (z.B. Pariboy ${ }^{\circledR}$ ) verdampft werden. Ergänzend ist zudem der Einsatz biologischer Kombinationspräparate zur Schleimhautregeneration (z.B. Mucosa comp. ${ }^{\circledR}$, Euphorbium comp. ${ }^{\circledR}$ ) möglich.
In fortschreitenden Erkrankungsfällen sind jedoch i.d.R. zusätzlich wiederholte antibiotische Behandlungen erforderlich. Diese dienen einerseits einer Reduktion der Mykoplasmen, andererseits einer Therapie von Sekundärinfektionen, die mitunter für eine akute Verschlechterung des Zustandes verantwortlich sind. Hier sollten in erster Linie Breitbandantibiotika Anwendung finden, die auch eine Wirkung gegen Mykoplasmen besitzen. Eingesetzt werden können:

- Fluorchinolone (z.B. Enrofloxacin, $10 \mathrm{mg} / \mathrm{kg}$ 1-mal tgl. p.o. oder sc.; Marbofloxacin, $4 \mathrm{mg} / \mathrm{kg}$ 1-mal tgl. p. o. oder s.c.)

- Doxycyclin (5-10 mg/kg 1-mal tgl. p.o. oder s.c.)

- Oxytetrazyclin (10-20 mg/kg. 1-mal tgl. s.c.)

- Erythromycin (10 mg/kg. 2-mal tgl. p.o.)

Die Wirksamkeit dieser Chemotherapeutika ist - je nach beteiligtem Mykoplasmenstamm - jedoch sehr unterschiedlich. Aufgrund der fehlenden Möglichkeit einer Erregerisolierung muss die Wirksamkeit daher ausprobiert werden. Kommt es nicht innerhalb weniger Tage zu einer deutlichen Besserung der Symptome, wird das Antibiotikum umgestellt.

In jedem Fall empfiehlt sich bei klinisch manifesten Mykoplasmosen zudem der Einsatz von Bronchodilatatoren (z.B. Theophyllin, 3-5 mg/kg 2-mal tgl. p.o.

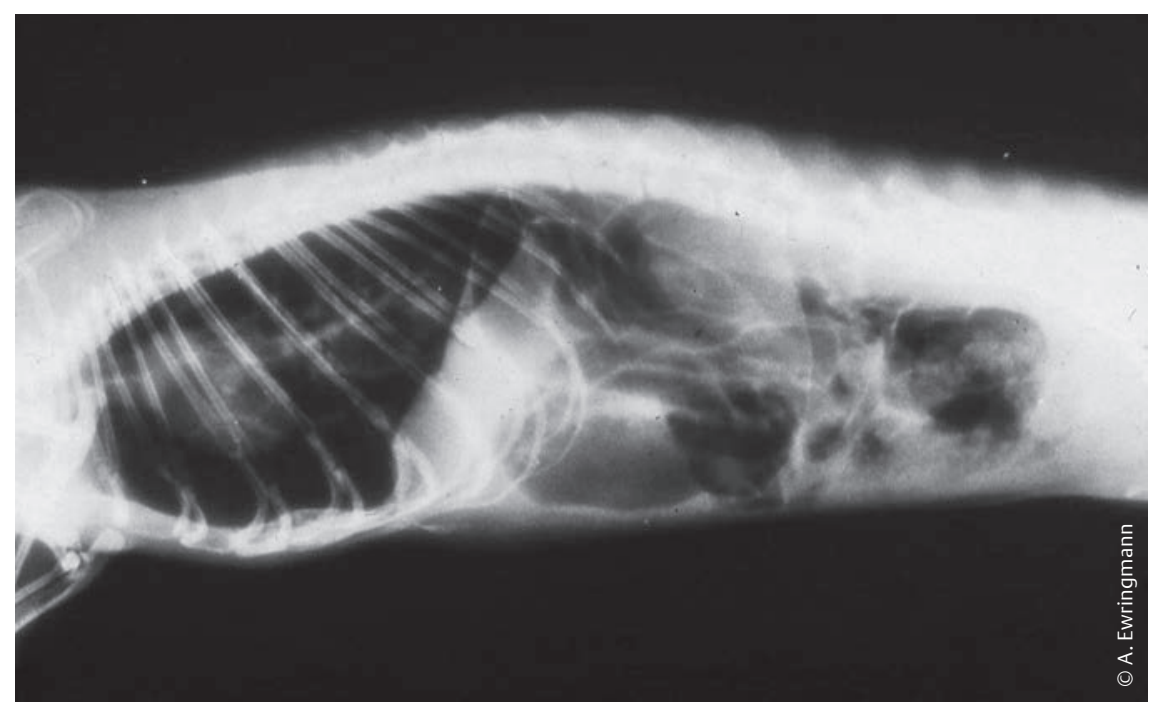

Abb. 9 Spontanpneumothorax bei fortgeschrittener Mykoplasmose. 


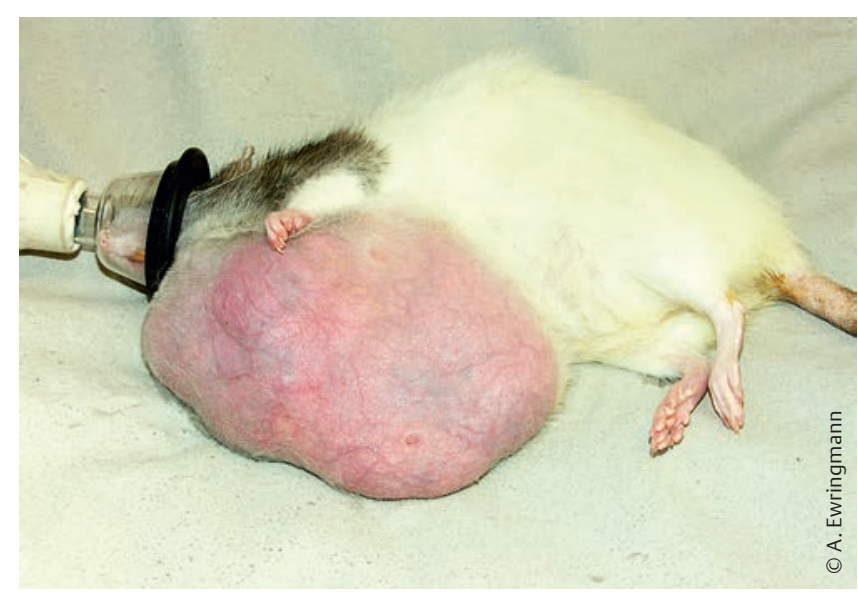

Abb. 10 Ausladendes Fibroadenom.

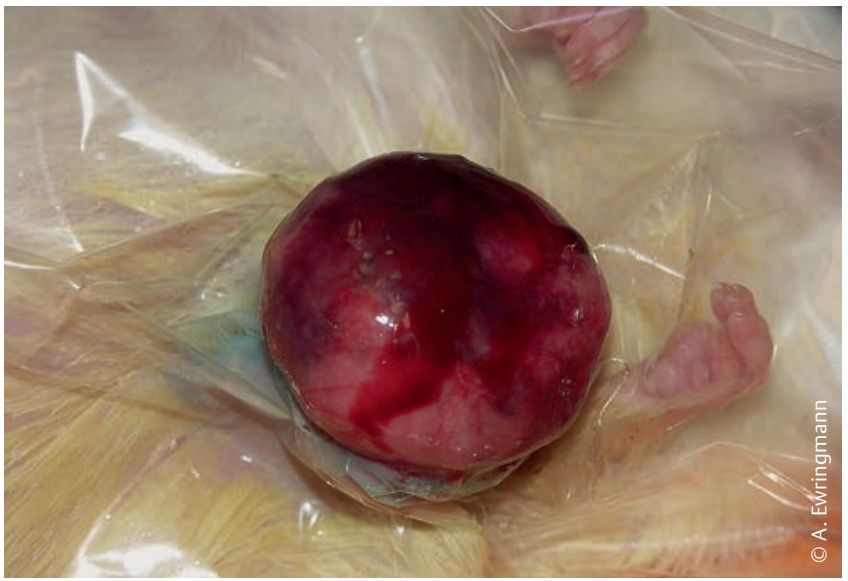

Abb. 11 Operationssitus eines Fibroadenoms. oder s.c.), um den Patienten die Atmung zu erleichtern. Diese sollten bei fortgeschrittener Erkrankung dauerhaft verabreicht werden.

Wichtig bei der Behandlung der Mykoplasmose ist zudem ein Ausschluss von Stressoren. Daher sollten detailliert die Haltungs- und Fütterungsbedingungen hinterfragt werden. Insbesondere muss die Art der Einstreu überprüft werden. Es empfiehlt sich zudem eine parasitologische Untersuchung von Sammelkotproben, um latente Endoparasitosen diagnostizieren und behandeln zu können.

\section{Neoplasien des Gesäuges}

Neoplasien des Gesäuges werden bei Ratten gehäuft beobachtet. Sie entstehen durch dauerhaften Östrogeneinfluss v.a. bei älteren Tieren. Auch eine genetische Prädisposition scheint eine Rolle zu spielen, da Mammatumore in manchen Familien gehäuft auftreten. Bei den meisten Umfangsvermehrungen handelt es sich um Fibroadenome, nur ca. 10-15\% sind Karzinome oder Adenokarzinome.

\section{Symptomatik}

Mammatumore werden als subkutane Umfangsvermehrungen an unterschiedlichen Lokalisationen sichtbar. Aufgrund des ausladenden Drüsengewebes, können die Tumore bis weit in die Inguinalregion sowie in den Achsel-, Nacken- und Vorderbrustbereich reichen. Die Neoplasien wachsen oft sehr schnell, erreichen mitunter enorme Größen und führen dadurch zu Bewegungseinschränkungen ( $\triangleright$ Abb. 10).
Die üblichen Fibroadenome sind palpatorisch meist gut begrenzt, relativ weich, mit einer glatten Oberfläche ( $\triangleright$ Abb. 11). Infiltrativ wachsende Adenokarzinome haben meist eine deutlich derbere Konsistenz und eine höckerige Oberfläche. Zudem ist eine palpatorische Abgrenzung oft nicht möglich. Eine Absicherung der Diagnose kann durch Punktion und zytologische Untersuchung erfolgen.

\section{Therapie und Prognose}

Bei malignen Neoplasien ist die Prognose als schlecht einzustufen. Sie neigen zur Metastasierung in die Lunge und zu schneller Rezidivbildung nach Exstirpation.

Fibroadenome können meist relativ einfach chirurgisch entfernt werden. Hierbei erfolgt nur eine Exstirpation der Neoplasie und des angrenzenden, unverändert aussehenden Drüsengewebes.

\section{.konkret}

Eine vollständige Entfernung der gesamten Milchleiste ist bei der Ratte wegen der speziellen anatomischen Verhältnisse nicht möglich.

Es besteht nach erfolgreicher Operation allerdings die Möglichkeit, dass es später andernorts (auch in eng benachbarten Regionen) zu erneutem Tumorwachstum kommt. Da auch die benignen Neoplasien oft sehr stark durchblutet sind, besteht bei Entfernung besonders großer Tumoren die Gefahr eines Kreislauf- zusammenbruchs durch übermäßigen Volumenverlust. Es ist daher anzuraten, Umfangsvermehrungen zu operieren, sobald sie beginnen, die Bewegungsfähigkeit des Tieres einzuschränken.

Da bei Ratten ein Tumorwachstum des Drüsengewebes durch Östrogene gefördert wird, kann eine frühzeitige Kastration weiblicher Tiere das Auftreten deutlich reduzieren.

\section{Pyometra}

Infektiöse Entzündungen der Gebärmutter kommen insbesondere bei älteren Ratten sehr häufig vor.

Keime können sich im Rahmen von Allgemeininfektionen in der Metra ansiedeln. Hier spielen v.a. Mykoplasmen eine große Rolle, die chronische Endometritiden mit Unfruchtbarkeit hervorrufen. Es besteht jedoch auch die Möglichkeit einer aufsteigenden Infektion über die Scheide. Diese ist normalerweise außerhalb der Brunst durch eine epitheliale Membran verschlossen. Bei älteren Tieren kommt es durch hormonelle Imbalancen oft nicht mehr zum vollständigen Verschluss der Scheidenöffnung, sodass Krankheitserreger eindringen können.

\section{Symptomatik}

Die ersten Anzeichen einer Infektion der Gebärmutter sind oftmals eine Schwäche in der Hinterhand und ein leicht schwankender Gang; manche Ratte laufen mit leicht aufgekrümmtem Rücken. Die Tiere sind weniger mobil und fressen schlechter. Es besteht in aller Regel eine 


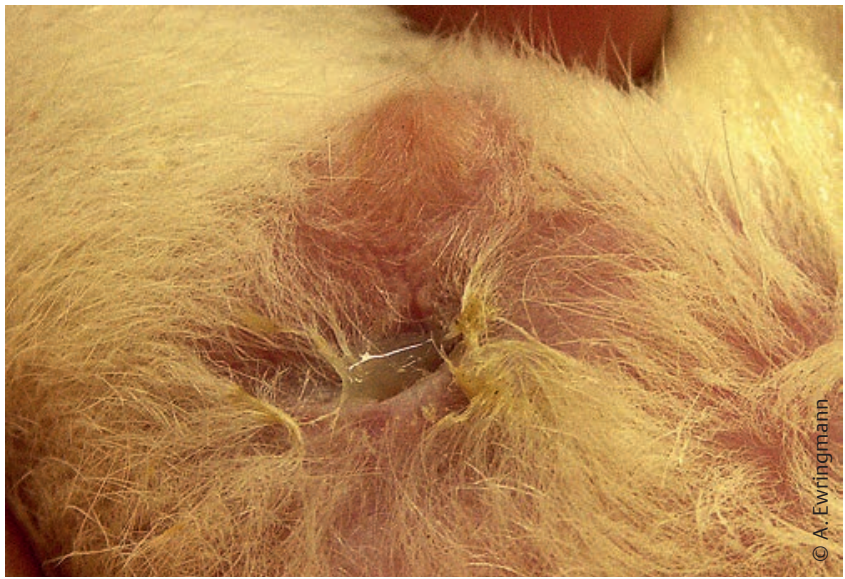

Abb. 12 Eitriger Scheidenausfluss bei Pyometra.

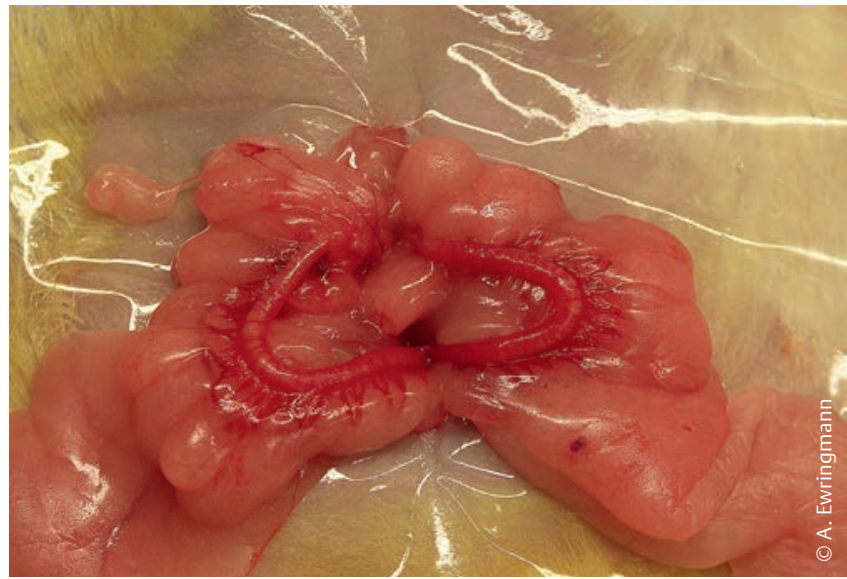

Abb. 13 Operationssitus bei Ovariohysterektomie: da eine offene Pyometra vorliegt, ist der Uterus kaum vergrößert. Auffällig sind die ausladenden Fetteinlagerungen im Lig. latum uteri, die bei gut genährten Ratten stets vorzufinden sind. offene Pyometra mit eitrigem oder blutig-eitrigem Vaginalausfluss, der von den Besitzern der Kleinnager oft erst sehr spät bemerkt wird, wenn sich die Tiere das Sekret nicht mehr wegputzen und beginnen, unangenehm zu riechen. In diesem Stadium weisen die Tiere meist ein deutlich reduziertes Allgemeinbefinden mit Apathie und Inappetenz auf. Oft besteht gleichzeitig eine beidseitige eitrige Konjunktivitis.

Der Uterus ist palpatorisch meist nicht vergrößert, kann allerdings eine deutliche Druckdolenz aufweisen. Durch Palpation des Abdomens bzw. der Gebärmutter lässt sich meist ein eitriger Vaginalausfluss induzieren ( $\bullet$ Abb. 12). Sonografisch lassen sich mit Flüssigkeit gefüllte Metraschlingen nachweisen.

\section{Therapie und Prognose}

Bei noch ungestörtem Allgemeinbefinden und erhaltener Futteraufnahme ist die Prognose für den Patienten sehr gut. Sie muss umso vorsichtiger gestellt werden, je schlechter der Allgemeinzustand ist.

Eine rein medikamentelle Behandlung ist i.d.R. nicht geeignet, um eine Infektion der Gebärmutter mit dauerhaftem Erfolg zu therapieren, da in dem Hohlorgan keine ausreichenden Antibiotikaspiegel erzielt werden können. Bei sehr alten oder instabilen Patienten, oder falls der Besitzer einer Operation nicht zustimmt, kann jedoch eine konservative
Therapie versucht werden, wenn eine offene Pyometra vorliegt.

Die Tiere werden zunächst durch Infusionen (Vollelektrolytlösung, 40-100 ml/ kg/d s.c., ggf. mit Glukosezusatz, bis zu $500 \mathrm{mg} / \mathrm{kg} / \mathrm{d}$ s.c.) stabilisiert. Sie erhalten zudem umgehend ein Antibiotikum (z.B. Sulfadoxin/Trimethoprim, 40/8 mg/ kg 2-mal tgl. p.o.; Enrofloxacin, $10 \mathrm{mg} / \mathrm{kg}$ 1-mal tgl. p.o. oder s.c.) und ein Analgetikum (z.B. Meloxicam, 0,15-0,5 mg/kg 1-2-mal tgl.p.o. oder s.c.); inappetente Patienten sind zwangszufüttern. Auch Aglepriston $\left(\right.$ Alizin $^{\circledR}$ ) kann beim Kleinsäuger angewendet werden. Es wird in einer Dosierung von $10 \mathrm{mg} / \mathrm{kg}$ 2-malig im Abstand von 24 Stunden subkutan appliziert. Mit einem feinen Katheter oder einer dünnen Ernährungssonde kann die Gebärmutter zusätzlich über die Scheide gespült werden. Zu diesem Zweck können physiologische Kochsalzlösung oder milde Antiseptika (z.B. Rebosan ${ }^{\circledR}$ ) verwendet werden. Nach der Spülung wird über die Sonde verdünnte antibiotische Injektionslösung in den Uterus instilliert. Der Therapieerfolg sollte nach einer Woche mittels Ultraschalluntersuchung überprüft werden.

Dauerhafte Erfolge sind meist nur durch eine Ovariohysterektomie ( $\triangleright$ Abb. 13) zu erzielen. Auch nach dem chirurgischen Eingriff ist eine intensive Betreuung, ggf. mit Zwangsernährung, erforderlich. Eine antibiotische Therapie ist für mindestens
1 Woche aufrechtzuerhalten. Ein Analgetikum sollte zumindest über 3-4 Tage appliziert werden.

\section{Online zu finden unte}

http://dx.doi.org/10.1055/s-0041-110694

\section{Literatur}

1 Brown C, Donnelly TM. Disease Problems of small rodents. In: Ferrets, rabbits, and rodents - Clinical medicine and surgery. Quesenberry KE, Carpenter JW, eds. 3rd ed. Elsevier; 2012: 354-372

2 Goodman G. Respiratory and cardiovascular disorders. In: BSAVA Manual of rodents and ferrets. Keeble E, Meredith A, eds. British Small Animal Veterinary Association; 2009: 142-149

3 Greenacre CB. Spontaneous tumors of small mammals. Vet Clin North Am Exot Anim Pract 2004; 7: 627-651

4 Hoefer H, Latney L. Urogenital and reproductive system disorders. In: BSAVA Manual of rodents and ferrets. Keeble E, Meredith A, eds. British Small Animal Veterinary Association; 2009: 150-160

5 Kling MA. A review of respiratory system anatomy, physiology, and disease in the mouse, rat, hamster, and gerbil. Vet Clin North Am Exot Anim Pract 2011; 14: 287-337

\section{Dr. Anja Ewringmann}

Praxis für kleine Heimtiere

Potsdamer Str. 1

12205 Berlin 